\section{Impact of Helicobacter pylori resistance in unsuccessfully pluritreated patients in a Department of Infectious Diseases in Rome}

Maria Teresa Mascellino, Barbara Porowska, Rosa Nicosia, Alessandra Oliva, Priscilla Boccia, Carola Severi

Department of Infectious Diseases, Policlinico Umberto I, Rome, Italy

\section{Abstract}

Twenty-five pluritreated patients were examined. Fifty-six percent yielded Helicobacter pylori (H. Pilory); of these, 9 patients showed a concomitant colonization of the three gastric regions.

The highest resistance rate was found for metronidazole $(71.8 \%)$ followed by chlaritromycin (53.1\%). Amoxycillin showed the best susceptibility (only 6\% of resistance), tetracycline showed $12 \%$ of resistant strains and levofloxacin appeared to be a promising antibacterial agent (18\% of resistance). The E-test method was shown to be more suitable than disk diffusion technique for resistance testing. Combined resistance to both chlaritromycin and metronidazole appeared in $50 \%$ of the strains. The isolates showing this dual resistance are known to be difficult to eradicate.

Resistotypes were shown to be genotypically different even if the strains with the resistance to both chlaritromycin and metronidazole are more likely to belong to genotype cagA+ and vacA slm1. Heteroresistance (different susceptibility of the isolated strains in a single stomach) resulted in $36 \%$ of patients with pangastritis. Indeed, the concomitant presence of H. pylori strains in the same subject, either susceptible or resistant or vice versa, may interfere with the eradication outcomes. In our study, antibiotic resistant $H$. pylori typically develops from pre-existing susceptible strains rather than from co-infection with a different and unrelated strain. In fact, each pair of isolates detected in our 4 patients with heteroresistance belonged to the same genotype (cagA+s $1 \mathrm{~m} 2$ in patient 1 and cagA+ slm1 in patients 2, 3 and 4).

In conclusion, $H$. pylori antibiotic resistance does present several issues in pluritreated patients owing to the rapid emergence of multi-resistant strains.

\section{Introduction}

Treatment regimens for $H$. pylori that have been used over the past decade are declining in efficacy and the treatment of $H$. pylori infection is bedevilled by drug-resistant strains. The leading causes of treatment failure are antimicrobial resistance and non-adherence to therapy. H. pylori is a microorganism which can easily acquire resistance to antimicrobial agents. Antibiotic resistance in bacteria can be categorized as intrinsic or acquired resistance: the first is a genetic property of most bacterial strains and typically evolves independently on the clinical use of antibiotics, the latter implies that a susceptible organism has developed resistance to antimicrobial agents to which it was previously susceptible. ${ }^{1-3}$

Antimicrobial susceptibility testing has, therefore, been proposed as a logical first step in treatment failure but controlled trials suggested that it may not always be essential for clinical management. ${ }^{4-10}$ European guidelines recommend performing susceptibility tests only before a third-line regimen or choosing "rescue" therapy. ${ }^{11-14}$ Infections in clinical trials, even with correct use of drug combinations, are not eradicated in $10-20 \%$ of patients; in clinical practice this percentage can be even higher. ${ }^{15-17}$

The patterns of resistance to antimicrobials may change with time, considering that in countries where chlaritromycin (CLA) resistance is progressively higher, the use of metronidazole (MZ)-based therapies is introduced, leading to subsequent MZ-resistance. ${ }^{18}$ Moreover, the in vitro results do not often correlate with in vivo efficacy. ${ }^{16,19}$

Recently it was also reported that multiple strains can colonize within a single stomach with differences in genotype distribution between different gastric locations ${ }^{10}$ as well as differences in minimum inhibitory concentrations of isolated $\mathrm{H}$. pylori.$^{20}$ Data on heteroresistance are, however, controversial, indicating that no single biopsy site can be considered representative of antimicrobial susceptibility testing. ${ }^{2,20,21}$

The aim of the present study was to evaluate the state of antimicrobial resistance, the eventual correlation of the susceptibility patterns with the strain genotype and the possible presence of heteroresistance in patients with previous multiple unsuccessful $H$. pylori eradication treatments.

\section{Materials and Methods}

The study population consisted of 25 consecutive out-patients, aged between 22 and 75, to whom at least two eradication regimens for $H$.
Correspondence: Maria Teresa Mascellino,

Department of Infectious and Tropical Diseases, Policlinico Umberto I, viale del Policlinico 155 , 00161, Rome, Italy.

E-mail: mariateresa.mascellino@uniroma1.it

Key words: Helicobacter pylori, susceptibility testing, strains genotyping, heteroresistance.

Contributions: MTM, RN, AO, PB, identification of microorganisms, isolation of the strains for testing antibiotic susceptibility and PCR for strains genotyping.

CS and BP, gastroduodenoscopy for drawing biopsies for $H$. pylori culture, histological examination and Urea Breath test.

Received for publication: 5 May 2010.

Accepted for publication: 22 June 2010.

This work is licensed under a Creative Commons Attribution 3.0 License (by-nc 3.0).

(C) Copyright M.T. Mascellino et al., 2010

Microbiology Research 2010; 1:e3

doi:10.4081/mr.2010.e3

pylori infection had failed. All patients had a persistently positive ${ }^{3} \mathrm{C}$-urea breath test (UBT). They underwent upper endoscopy with biopsies for $H$. pylori culture, susceptibility testing and histological evaluation. The patients were recruited at the Policlinico Umberto I Academic Hospital of the University of Rome "La Sapienza". All patients were asked to give informed consent for undergoing an oesophago-gastroduodenoscopy with multiple biopsies. This independent study was approved by the local Ethics Committee and was not sponsored by any pharmaceutical company. Patients were excluded in case of gastric surgery, malignant disease, pregnancy or lactation and atrophic body gastritis. Intake of antibiotics, PPI, bismuth or $\mathrm{H}_{2}$-antagonists were interrupted during the four weeks before endoscopy.

\section{Bacterial culture and susceptibility testing}

Biopsies for culture (3 samples from the antrum, 3 samples from the corpus and 3 samples from the fundus) were first obtained and then collected into 3 separate sterile containers containing $1 \mathrm{cc}$ of sterile saline solution. Samples for culture study were sent to the microbiological laboratory within three hours from sampling. All biopsies were urease-positive. The culture test was performed separately on gastric biopsy specimens drawn from the different sites. Specimens obtained from a single gastric region were pooled together for culture. Essential conditions for the $H$. pylori growth were the following: microaerophilic 
atmosphere, temperature $37^{\circ}$ (range $33-40^{\circ}$ ), presence of $0.5 \%$ glycine. The culture media used were: a) blood agar Columbia with addition of cyclodextrane, 10\% of horse blood, antibiotics and haemine; b) Pylori Selective agar (bio-Merieux) with 5\% of sheep blood and antibiotics (amphotericin, vancomycin and trimethoprim). The identification of the microorganisms was performed through the following tests: colony morphology, characteristic spiral-shaped, Gram-negative bacteria and positive findings on oxydase, urease and catalase tests.

Once the colonies were identified as $H$. pylori in the primary isolation, a sub-culture was performed in order to obtain a secondary isolation used for antibiotic sensitivity tests, for the strain typing and strain preservation. The methods used for antimicrobial agents susceptibility testing were Kirby-Bauer technique and E-test. The antibiotics tested were: metronidazole (MZ), levofloxacin (LEV), tetracycline (TE), chlarithromycin (CLA) and amoxycillin (AMX).

Modified Kirby-Bauer disk diffusion method $(\mathrm{K}-\mathrm{B})^{22}$ was performed by preparing a standard inoculum equivalent to 2 MacFarland of fresh culture of $H$. pylori in Brain Heart Infusion broth (BHI Becton-Dickinson), inserting two antibiotic disks for each plate. For K-B method, the inhibition halos were interpreted following the data in literature. ${ }^{23}$ The strains were considered resistant if the inhibition halos were: $\leq 16 \mathrm{~mm}$ for $\mathrm{MZ}, \leq 18 \mathrm{~mm}$ for AMX and $\leq 30 \mathrm{~mm}$ for CLA, LEV and TE.

For E-test procedure, Mueller-Hinton agar with $5 \%$ sheep blood was used as base medium. The plates were streaked in three directions with each inoculum to produce a lawn of bacterial growth. E-test strips were aseptically placed onto the dried surfaces of the inoculated plates. The E-test plates were incubated under $12 \% \mathrm{CO}_{2}$ at $37^{\circ} \mathrm{C}$. In order to define the strain resistance with the E-test method, the following break-points were used: MIC $\geq 2$ $\mu \mathrm{g} / \mathrm{mL}$ for AMX and CLA, MIC $\geq 4 \mu \mathrm{g} / \mathrm{mL}$ for TE, $\mathrm{MIC} \geq 8 \mu \mathrm{g} / \mathrm{mL}$ for MZ and LEV. ${ }^{24,25}$

For both methods, the borderline values were interpreted as intermediate strains (i.e. for MZ the intermediate values were considered as follows: inhibition halos between 16 and $21 \mathrm{~mm}$ for K-B method and MIC between 6 and 8 $\mathrm{mcg} / \mathrm{mL}$ for E-test). ${ }^{26}$ Two quality control reference strains were used throughout the testing: H. pylori ATCC43504 and H. pylori RD26.

\section{Strain genotyping}

Genomic DNA was extracted from sweep cultures of $H$. pylori, and the primers and PCR conditions for the assay for cagA (a marker for the 3 ' end of the cag pathogenicity island and for the cagI region), using the D008/R008 primer set, were as described previously. ${ }^{27}$ Vacuolating cytotoxin (vacA) genotyping based on signal (s)-and mid (m)-region alleles was performed using a multiplex assay. This method is able to genotype $H$. pylori isolates based on the main virulence genes (cag and vac). Briefly, biopsies collected in Eppendorf tubes containing $500 \mathrm{mcl}$ of sterile phosphate buffered saline, were vortexed vigorously for 2 min. The tubes were then boiled in a water bath for $15 \mathrm{~min}$, cooled on ice and centrifuged for $1 \mathrm{~min}$ at $13,000 \mathrm{~g}$. The supernatant was transferred to another tube with $1 \mathrm{mcl}$ of the template for amplification. Multiplex PCR was carried out in $25 \mathrm{mcL}$ volumes using $10 \mathrm{ng}$ of DNA, $1 \mathrm{U}$ of Taq polymerase, 10 pmol of both oligonucleotide primers of all the selected genes per reaction, $0.25 \mathrm{mmol} \mathrm{l}^{-1}$ deoxynucleotide triphosphate and $2-3 \mathrm{mmol}^{-1} \mathrm{MgCl}_{2}$ in standard PCR buffer for 35 cycles: initial denaturation at $95^{\circ} \mathrm{C}$ for $5 \mathrm{~min}, 35$ cycles of denaturation at $94^{\circ} \mathrm{C}$ for $30 \mathrm{sec}$, annealing at $52^{\circ} \mathrm{C}$ for $1 \mathrm{~min}$, extension at $72^{\circ} \mathrm{C}$ for $1 \mathrm{~min}$, followed by final extension at $72^{\circ} \mathrm{C}$ for $7 \mathrm{~min}$. The strain ATCC 49503 was used as positive control.

PCR products were electrophoresed in agarose gel with $0.3 \%$ of ethidium bromide in a 10\% Tris-borate-EDTA buffer. Gel was visualized under a UV transilluminator. ${ }^{28}$

\section{Statistics}

Fisher's exact test and $P$ values were determined. $\mathrm{P}<0.05$ was considered statistically significant.

\section{Results}

A total of 25 patients (20 females and 5 males; median age 49 years; range 22-75 years) with $H$. pylori positive gastritis were included in the study. All patients were positive by histopathology and urease tests. The median number of previous eradication treatments was 3 (range 2-9).

Out of 75 specimens (25 of which were taken from antrum, 25 from corpus and 25 from fundus), all of them taken from the 25 patients enrolled, 35 strains of $H$. pylori were isolated in 14 subjects (56\%). Of these strains, 13 were detected in antrum, 11 in corpus and 11 in fundus (in one patient $H$. pylori was found in the corpus and fundus but not in the antrum). The growth time required was approximately one week, although after three days few colonies could be detected. However, since patients in the present study had already been treated in the past with multiple antibiotic therapies, culture media were incubated up to 14 days in order to achieve optimal growth.

H. pylori colonies appeared as small, gray, translucent, associated dots.

The susceptibility tests with both methods were performed in 32 out of 35 strains, due to the transformation into coccoid forms of $3 H$. pylori strains belonging to 3 specimens taken from the different gastric regions of the same patient.

For the K-B method, MZ showed the highest resistance rate (21/32 strains) followed by CLA (17/32). Resistance to LEV was found in 6 out of 32 strains and that to TE in 4 out of 32 . AMX showed the lowest resistance rate (2/32) (Table 1).

MICs of the 5 antimicrobial agents were obtained by the E-test method (Table 2).

Twenty-three out of 32 strains resulted resistant to MZ (MIC $\geq 8 ; 71.87 \%$ ), with 3 isolates having MICs $\geq 256$.

Seventeen strains had MIC $\geq 2$ for CLA (53.12\%) and only one strain showed MIC $\geq 256$.

For TE, AMX and LEV, most strains were included in the range 0.5-1.5 and none showed MIC $\geq 48$. Resistance rates were $6.25 \%$ for AMX, 12.51\% for TE and 18.75\% for LEV (Tables 1 and 2). No discrepancies were observed indeed between the two methods used (E-test and Kirby-Bauer) regarding antibiotic susceptibility testing, except for MZ, as 2 strains resulted to be resistant only by E-test (23 towards 21) (Table 2).

Combined resistance for up to two antibiotics was found in almost $43.74 \%$ of the strains (9.37\% with only one resistance and $34.37 \%$ with two resistances) while only $21.87 \%$ was susceptible to all antibiotics (Figure 1).

Each isolate of $H$. pylori was characterized by the assignment of a susceptibility pattern based on its combined susceptibilities or resistances to MZ or CLA (Table 3). Overall, $21.8 \%$ of strains were fully sensitive (MZ-susceptible and CLA-susceptible) whereas 50\% (16 strains) were resistant to both antibiotics having MIC for MZ $\geq 8$ and MIC for CLA $\geq 2$ contemporaneously. The resistances to both CLA and MZ combined to LEV or to TE were analyzed separately. Combined resistance to $\mathrm{MZ}$, CLA and LEV was found in $18.75 \%(6 / 32)$ of strains, while resistance to MZ, CLA and TE was found in $9.37 \%(3 / 32)$. The only two strains resistant to AMX were resistant to both CLA and MZ but not to TE or LEV (data not shown).

Genotypes (cagA status and vacA allelic form) were determined for 28 isolates (16 MZresistant and CLA-resistant, 7 CLA-susceptible and MZ-susceptible, 5 MZ-resistant and CLAsusceptible. The four intermediate strains have not been considered. No strains were found for the group MZ-susceptible (S) and CLA-resistant (R). The numbers of the strains grouped by antibiotic-susceptibility pattern and combined genotypes are shown in Table 4.

Most isolates were cagA-positive (22/27; $78.5 \%)$ and these were either vacA type s1m1 (63\%) or slm2 (31.8\%), with only one isolate that was $\mathrm{s} 2 \mathrm{~m} 2$. For the cagA-negative isolates, 
the vacA $m 2$ form was a feature of most (4/6) isolates, of which $3(75 \%)$ were vacAs1m2. Within the group the two predominant susceptibility Patterns (MZ-resistant and CLA-resistant or MZ-susceptible and CLA-susceptible) which represented $82 \%$ of isolates, were genotypically diverse. High-level resistance to either MZ or CLA was not associated with a particular vacA genotype as most strains, irrespective of resistotype, had the vacA s1 allele (i.e. 6/7 of MZ-S and CLA-S isolates). The distribution of the mid-region alleles was more variable. Overall, $66 \%(8 / 12)$ of the $\mathrm{m} 2$ isolates and $81 \%(13 / 16)$ of the $\mathrm{ml}$ isolates resulted resistant to MZ. Within the group of patients affected by pangastritis (19/25, 76\%), in 11 subjects where $H$. pylori strains were isolated, 9 (81.8\%) showed a concomitant colonization of the 3 gastric regions. Four patients out of 11 (36.3\%) showed a different pattern of antibiotic sensitivity/resistance (heteroresistance) of $H$. pylori isolates in various gastric regions (antrum and corpus-fundus) (Table 5). In 3 patients, $H$. pylori strains in the antrum were CLA-susceptible whereas those in the corpusfundus were resistant; similar heteroresistance (susceptible in the antrum and intermediate or resistant in the corpus-fundus) was observed in 2 patients for AMX and for MZ, respectively. Patient 3 showed a double change of sensitivity concerning both MZ and CLA (Table 5).

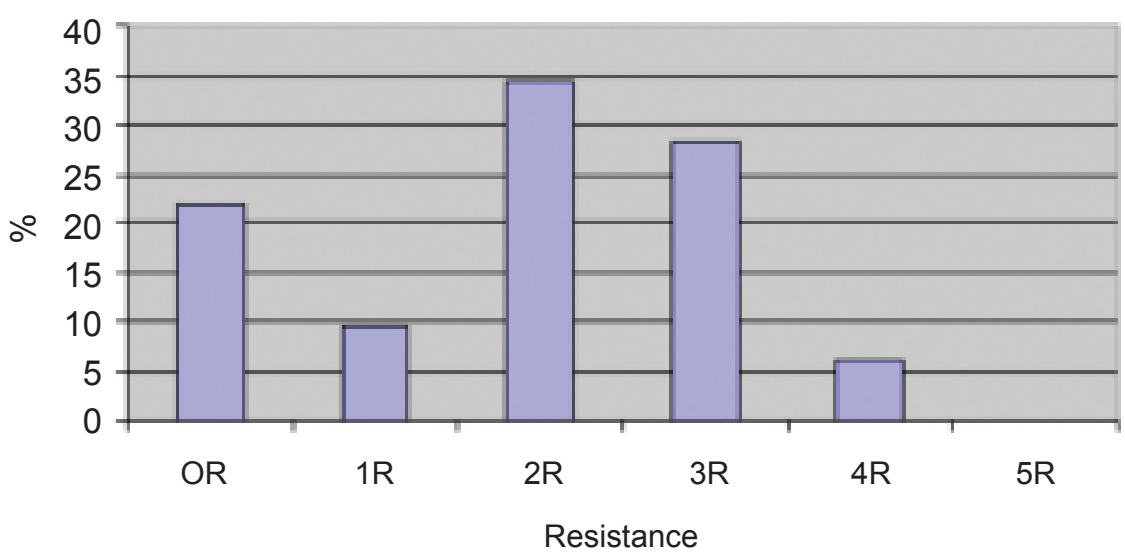

$0 R$, strains with no resistances detected; $1 R$, strains with resistance to only one antibiotic; $2 R$, strains with resistance to two antibiotics contemporaneously; $3 \mathrm{R}$, strains with resistance to three antibiotics contemporaneously; $4 \mathrm{R}$, strains with resistance to four antibiotics contemporaneously; $5 \mathrm{R}$, strains with resistance to five antibiotics contemporaneously.

Figure 1. Percentage of $H$. pylori strains with different combinations of resistance.

Table 1. Susceptibility tests of $H$. pylori strains isolates with K-B method. Total strains: 32.

\begin{tabular}{|c|c|c|c|c|c|}
\hline & $\begin{array}{c}\text { MZ } \\
\%(\mathbf{N})\end{array}$ & $\begin{array}{l}\text { CLA } \\
\%(\mathrm{~N} .)\end{array}$ & $\begin{array}{l}\text { LEV } \\
\%(\mathrm{~N} .)\end{array}$ & $\begin{array}{c}\text { TE } \\
\%(\mathbf{N})\end{array}$ & $\begin{array}{c}\text { AMX } \\
\%\left(\mathrm{~N}^{\prime}\right)\end{array}$ \\
\hline Sensitive & $28.12(9)$ & 40.63 (13) & $81.25(26)$ & 78.12 (25) & $90.63(29)$ \\
\hline Intermediate & 6.25 (2) & $6.25(2)$ & $0(0)$ & $9.37(3)$ & $3.12(1)$ \\
\hline Resistant & $65.62(21)$ & 53.12 (17) & $18.75(6)$ & $12.51(4)$ & $6.25(2)$ \\
\hline
\end{tabular}

Table 2. Distribution of MIC values for $32 \mathrm{H}$. pylori isolates with E-test method. The number of intermediate strains is reported in brackets.

\begin{tabular}{|c|c|c|c|c|c|c|c|c|c|c|}
\hline $\begin{array}{l}\text { ANTIMICROBIAL } \\
\text { AGENTS }\end{array}$ & $\leq 0.5$ & $0.5-1.5$ & $2-3.5$ & $4-7.5$ & 8-32 & $48-128$ & $\geq 256$ & $\begin{array}{l}\text { MIC } \\
\text { cut off }\end{array}$ & $\begin{array}{l}\text { N. of resistant } \\
\text { strains (intermediate) }\end{array}$ & $\begin{array}{l}\text { Total } \\
\text { strains }\end{array}$ \\
\hline MZ & 0 & 2 & 4 & 3 & 16 & 4 & 3 & $\geq 8$ & $23+(2)=25$ & 32 \\
\hline CLA & 6 & 9 & 5 & 4 & 4 & 3 & 1 & $\geq 2$ & $17+(2)=19$ & 32 \\
\hline LEV & $\begin{array}{c}8 \\
32\end{array}$ & 10 & 4 & 4 & 6 & 0 & 0 & $\geq 8$ & $6+(0)=6$ & \\
\hline $\mathrm{TE}$ & 10 & 9 & 9 & 2 & 2 & 0 & 0 & $\geq 4$ & $4+(3)=7$ & 32 \\
\hline AMX & 17 & 13 & 2 & 0 & 0 & 0 & 0 & $\geq 2$ & $2+(1)=3$ & 32 \\
\hline
\end{tabular}

MZ, metronidazole; TE, tetracycline; CLA, chlaritromycin; AMX, amoxycillin; LEV, levofloxacin.

Table 3. Combined susceptibility and resistance of H.pylori to chlaritromycin, metronidazole and A) levofloxacin; B) tetracycline.

A) Susceptibility pattern

\begin{tabular}{|c|c|c|c|}
\hline CLA & MZ & LEV & N. of strains (\%) \\
\hline $\mathrm{R}$ & $\mathrm{R}$ & S & $10(31.25)$ \\
\hline $\mathrm{R}$ & $\mathrm{R}$ & $\mathrm{R}$ & $6(18.75)$ \\
\hline$S$ & S & S & 7 (21.87) \\
\hline $\mathrm{R}$ & S & S & $0(0)$ \\
\hline $\mathrm{R}$ & S & $\mathrm{R}$ & $0(0)$ \\
\hline S & $\mathrm{R}$ & S & $5(15.62)$ \\
\hline
\end{tabular}

\begin{tabular}{|c|c|c|c|}
\hline CLA & MZ & TE & N. of strains (\%) \\
\hline $\mathrm{R}$ & $\mathrm{R}$ & S & $13(40.63)$ \\
\hline $\mathrm{R}$ & $\mathrm{R}$ & $\mathrm{R}$ & $3(9.37)$ \\
\hline S & S & S & $7(21.87)$ \\
\hline $\mathrm{R}$ & S & S & $0(0)$ \\
\hline $\mathrm{R}$ & S & $\mathrm{R}$ & $0(0)$ \\
\hline S & $\mathrm{R}$ & S & $2(6.25)$ \\
\hline$\underline{S}$ & $\mathrm{R}$ & $\mathrm{R}$ & $2(6.25)$ \\
\hline
\end{tabular}


Table 4. Distribution of $\boldsymbol{H}$. pylori by genotype and antibiotic susceptibility pattern.

\begin{tabular}{|c|c|c|c|c|}
\hline \multirow[b]{2}{*}{ Strain genotype } & \multicolumn{4}{|c|}{ N. of strains by resistotype } \\
\hline & $\begin{array}{l}\text { MZS-CLAS } \\
\text { (7 strains) }\end{array}$ & $\begin{array}{c}\text { MZR-CLAR } \\
\text { (16 strains) }\end{array}$ & $\begin{array}{l}\text { MZR-CLAS } \\
\text { (5 strains) }\end{array}$ & $\begin{array}{l}\text { Total } \\
\text { (28) }\end{array}$ \\
\hline cagA+ slm1 & 2 & 10 & 2 & 14 \\
\hline cagA+ slm2 & 2 & 5 & 0 & 7 \\
\hline cagA+s2m2 & 0 & 0 & 1 & 1 \\
\hline cagA-slml & 1 & 1 & 0 & 2 \\
\hline cagA- slm2 & 1 & 0 & 2 & 3 \\
\hline cagA-s2m2 & 1 & 0 & 0 & 1 \\
\hline
\end{tabular}

No strains were found in the resistotype MZS-CLAR. MZS, metronidazole-susceptible; MZR, metronidazole-resistant; CLAS, chlaritromycinsusceptible; CLAR, chlaritromycin-resistant.

Table 5. Heteroresistance of $\mathrm{H}$. pylori isolates to antibiotics in 4 patients, in three different districts of the stomach and related genotypes.

\begin{tabular}{|c|c|c|c|c|}
\hline & Patient 1 & Patient 2 & Patient 3 & Patient 4 \\
\hline $\begin{array}{l}\text { CLA } \\
\text { Strains } \\
\text { genotype }\end{array}$ & $\begin{array}{c}\mathrm{S}(\mathrm{A}) \rightarrow \mathrm{R}(\mathrm{C}) \\
\text { cagA+ slm2 }\end{array}$ & & $\begin{array}{c}\mathrm{S}(\mathrm{A}) \rightarrow \mathrm{I}(\mathrm{C}) \\
\mathrm{cagA}+\mathrm{s} l \mathrm{l} l\end{array}$ & $\begin{array}{c}\mathrm{S}(\mathrm{A}) \rightarrow \mathrm{R}(\mathrm{C}) \\
\mathrm{cagA}+\mathrm{s} \operatorname{lm} 1\end{array}$ \\
\hline $\begin{array}{l}\text { AMX } \\
\text { Strains } \\
\text { genotype }\end{array}$ & & $\begin{array}{c}\mathrm{S}(\mathrm{A}) \rightarrow \mathrm{I}(\mathrm{C}) \\
\text { cagA + slml }\end{array}$ & & \\
\hline $\begin{array}{l}\text { MZ } \\
\text { Strains } \\
\text { genotype }\end{array}$ & & & $\begin{array}{c}\mathrm{S}(\mathrm{A}) \rightarrow \mathrm{R}(\mathrm{C}) \\
\text { cagA }+ \text { slml }\end{array}$ & \\
\hline
\end{tabular}

C, corpus-fundus; A, antrum; S, susceptible; R=, resistant; I, intermediate CLA, chlaritromycin; AMX, amoxycillin; MZ, metronidazole.

The five pairs of the strains showing different sensitivity to CLA, MZ and AMX in various stomach districts, were genotypically identical to each other: in patient \#1, both strains belonged to the genotype cagA+s1m2 whereas each pair from the other 3 patients belonged to the same genotype cagA+ slml (Table 5).

\section{Discussion}

Helicobacter pylori eradication continues to be a challenge in a small group of patients after the failure of several therapeutic regimen attempts. After two courses of treatment, which generally include PPI-based triple and quadruple therapy regimens, about $4-6 \%$ of patients remained infected. ${ }^{14}$ Treatment regimen is generally chosen on the basis of the prevalence of bacterial resistance detected against the tested antibiotics; particularly chlarithromycin which still remains the most potent drug against this infection. Following the therapeutic guidelines, when CLA resistance is greater than 15-20\% and MZ-resistance greater than $40 \%$, a triple therapy is suggested for 14 days for the first-line treatment (IPP+CLA $500 \mathrm{mg}+\mathrm{AMX} 1 \mathrm{~g}$ or tinidazole 500 $\mathrm{mg}$ ) or for the second-line IPP+LEV $250 \mathrm{mg}+$ AMX $1 \mathrm{~g}$ for ten days (in case of AMX allergy, it is advisable to use CLA or tinidazole together with LEV). ${ }^{29}$

The question whether susceptibility testing can be helpful in guiding therapeutic strategies is still controversial. Some literature data show that a successful eradication can be achieved in almost all patients without susceptibility testing, $;, 30$ other data, on the other hand, state that even a first-line therapy should rather be scheduled on the basis of sensibility/resistance of $H$. pylori to antibiotics. ${ }^{8,25,31}$

Failure to eradicate may be due to non-compliance in some cases, but antibiotic resistance is recognized as a significant problem as indicated in various clinical trials ${ }^{26}$ and by the fact that post-treatment failures have a high rate of infection with resistant strains. ${ }^{32}$

In the present study $H$. pylori was detected in $56 \%$ of patients; a rate that is far below those reported by other ${ }^{33}$ This is probably due to the highly selected population consisting mostly of pluritreated dyspeptic patients with pangastritis. As a matter of fact, the chance of isolating this bacterium from the biopsies was probably influenced by the pathologies in the patients under study; $H$. pylori isolation was more complex in patients with dyspepsia or gastro-oesophageal reflux than in patients with ulcers. ${ }^{34}$ In patients with pangastritis (the main pattern of gastritis observed in the group under study), H. pylori infection is considered quite characteristic because the bacteria are able to colonize a stomach with reduced acid secretion, and also virulence and persistence mechanisms may be different with respect to patients with normal acid secretion, as already suggested by Blaser and Atherton. ${ }^{35}$ However, other factors may probably contribute to the low detection rate. First of all, in the present study, the isolation rate was likely influenced by the sole use of the culture technique. By overcoming the difficulty in accessing the $H$. pylori ecological niche and the fragile nature of the bacterium, ${ }^{36}$ non-culture methods (such as fluorescence in situ hybridization - FISH test) may be more sensitive than culture-based techniques. ${ }^{33}$

Since a perfect method for $H$. pylori isolation is not available and the methods used in each laboratory strongly affect its detection, two growing media were used and compared. $H$. pylori is, in fact, a fastidious microorganism to grow, requiring particular enriched culture media. The best medium for the primary isolation of $H$. pylori was the selective Pylori agar, which is more suitable for its detection mainly because it is more likely to avoid the transformation of vital germs into coccoid forms that are unable to grow and which would thus not be suitable for antibiotic sensitivity study. ${ }^{36,37}$ The low percentage of $H$. pylori isolated in our study (56\%), considering that all our patients were infected because they persistently resulted positive to both Urea Breath test and histological examinations, can also be due to the fact that they could yield only very low numbers of bacteria (too low to be cultured) owing to several previous treatments or to the presence of metabolic inactive microorganisms that are insensible to antibiotics.

When testing antibiotic sensitivity in vitro, both methods used (E-test and Kirby-Bauer) have given similar results, thus confirming previous considerations ${ }^{23,26}$ and yet highlighting a slight difference in MZ resistance which was higher with E-test. The disk-diffusion method is less reliable for those microorganisms (such as $\mathrm{H}$. pylori) that need a protracted incubation due to the pattern of the antibiotic release from the disks. On the contrary, the E-test has a more stable pattern of antibiotic release and seems to better tolerate an extended incubation time. The E-test might overestimate MZ resistance due to the presence of intermediate MIC levels not found on the Kirby Bauer scheme. ${ }^{23}$ In any case, in our study both methods showed good reproducibility.

The criteria for intermediate resistance and their clinical role have not yet been established. Data concerning this group are, in fact, controversial $^{26}$ and no defined standards were produced for identifying the category of low susceptible or low resistant isolates. From this point of view, MZ was the most studied antimicrobial agent. Intermediate susceptibility values (MIC $\geq 2$ to $\leq 8 \mu \mathrm{g} / \mathrm{mL}$ or $16-21 \mathrm{~mm}$ zone of growth inhibition) were, in fact, recorded for 
MZ, while for other antibiotics no approved standardized methods were developed ${ }^{26}$

Levofloxacin, often used in second-line therapeutic schedules, is considered a promising antimicrobial agent for $H$. pylori infection $\mathbf{s}^{38}$ and has proven to be a good alternative for therapyresistant infections. This is the reason why LEV in combination with other antibiotics should be considered. $^{25}$ The present study found LEV resistance in nearly $20 \%$ of patients. This confirms that after multiple treatments a development of resistance to recently introduced antibiotics may occur. This means that in the future new antibiotic molecules need to be considered in the treatment of $H$. pylori infection. The high rate of MZ resistance observed in our study is in line with the current data. ${ }^{39,40}$ Resistance to various antibiotics is increasing worldwide, especially in those countries where their use is extensive (MZ for example is widely used for gynecological infections and macrolides are frequently used in respiratory diseases). ${ }^{41,42}$ Furthermore, the extensive use of MZ in empirically-based therapy of early $H$. pylori infections and its re-administration in bismuth-based quadruple regimen after previous failure of MZ containing regimens, ${ }^{17}$ can explain the high rate of resistance as found in our study (71.87\%). Moreover, the microaerophilic atmosphere in which $H$. pylori grows can interfere with the activity of MZ which requires a strict anaerobic condition in vitro.

The antibiotic resistance rates observed in the present study were generally higher than those reported in naive patients, as well as those observed in patients who only underwent one unsuccessful eradication therapy. ${ }^{30}$ The high resistance rates observed may be related to the high number of administered therapy cycles (up to 9), suggesting that the repeated treatments increase antibiotic resistance. In fact, $H$. pylori is known to be a microorganism which can easily acquire resistance to antimicrobial agents. In our study, however, data concerning the possible presence of antibioticresistant strains prior to the administration of eradication therapies are not available because the first culture was performed after the second course of therapy had proven unsuccessful.

Another factor that could affect the efficacy of current therapeutic regimens is the occurrence of concomitant antibiotic resistance. $H$. pylori isolates resistant to both MZ and CLA are considered difficult to eradicate..$^{25}$ In our study, $50 \%$ of the isolated strains showed this combined resistance, supporting the difficulty in H. pylori eradication. Kist and Glocker ${ }^{43}$ concluded that repeated empirical treatment regimens were especially associated with posttreatment presence of strains exhibiting dual resistance to $\mathrm{MZ}$ and CLA. In our study, these strains, even if they cannot be strictly associated with any particular strain genotype, had high level MICs of greater than $256 \mu \mathrm{g} / \mathrm{mL}$ to MZ and such isolates could be viewed as potentially difficult to eradicate. Our analysis indicated that resistance to CLA cannot arise in MZ-susceptible strains. In fact, no MZ-S and CLA-R type was found, unlike the study of Elviss ${ }^{26}$ in which a small percentage of this type (3\%) was detected.

Combined resistance was also found for LEV and AMX associated with CLA and MZ resistance ( $18.75 \%$ and $6.25 \%$, respectively). No dual resistance was found for the AMX - TE combination. Yahav et al. ${ }^{25}$ emphasize a strong association between resistances to CLA and LEV and thus suggest not to include LEV in the triple therapy of patients whose isolates proved to be resistant to CLA.

Our strains were genomically diverse and there were no particular cag A or vacA forms associated with metronidazole resistance, even if this type can be more markedly correlated (but not statistically significant, $\mathrm{P}>0.5$ ) to the genotype cagA+ and vacA slm1 (a common genotype also in susceptible isolates) whereas the metronidazole susceptibility strains more often showed the genotype cagAand vacA s1m1 or $\mathrm{s} 1 \mathrm{~m} 2$ or $\mathrm{s} 2 \mathrm{~m} 2$. MZ resistance may be partially due to mutations in nitroreductase genes. ${ }^{26}$

Finally, also heteroresistance concerning a distinct pattern of antibiotic sensitivity of isolates belonging to different districts of the same stomach can interfere with therapeutic outcomes. ${ }^{41}$ Strain diversity proved to occur in different biopsies from the same individual. ${ }^{1,2,7,44,45}$ Heteroresistance to MZ has often been shown. Obra et al. ${ }^{1}$ found cultures containing mixed MZ-S and MZ-R isolates in 10\% of cases.

Considering the genetic relationship of the isolates showing heteroresistance, we can highlight that the MZ resistance can be due to ex novo mutations (acquired resistance) and not to the horizontal transfer of genes among unrelated strains. In our study, following the genetic typing of the pairs of strains showing different susceptibility patterns in various stomach districts, it can be deduced that antibiotic resistant $H$. pylori typically develops from pre-existing susceptible strains rather than from co-infection with a different strain. In fact, we demonstrated that each pair of isolates in our patients with heteroresistance belonged to the same genotype (cagA+ s1m2 in patient \#1 and cagA+ s1m1 in patients \#2, 3 and 4).

Yet other authors ${ }^{20}$ agree with the statement that an individual may have a mixed $H$. pylori infection with respect to a different antimicrobial susceptibility in various gastric regions.

Consequently, in order to avoid misclassifying a strain as sensible where only one biopsy region was investigated, heteroresistance between three biopsy sites from each patient should always be considered.

In conclusion, $H$. pylori antibiotic resistance state in pluritreated patients does present several aspects that, associated with the predominant pattern of gastritis, could interfere with the eradication outcomes. It is, therefore, important to continue monitoring antibiotic resistance in order to have accurate information on local rates to guide selection of the most specific and appropriate treatment regimens.

\section{References}

1. Obra P, de la Alarcon T, Domingo D, et al. Heteroresistance to metronidazole and genetic relationship of helicobacter pylori clinical isolates. Gut 2001. Epub ahead of print October 1.

2. Kim JJ, Kim JG, Kwon DH. Mixed-infection of antibiotic susceptible and resistant Helicobacter pylori isolates in a single patient and underestimation of antimicrobial susceptibility testing. Helicobacter 2003;8:202-6.

3. Yan WH, Chen J, Hu HJ, et al. Preliminary study on "in vitro" induction of antibiotic resistance in Helicobacter pylori strains isolated from children. Zhonghua $\mathrm{Er} \mathrm{Ke} \mathrm{Za}$ Zhi 2007;45:708-11.

4. Pilotto A, Franceschi M, Rassu M, et al. Incidence of secondary Helicobacter pylori resistance to antibiotics in treatment failures after 1-week proton pump inhibitorbased triple therapies: a prospective study. Dig Liver Dis 2000;32:667-72.

5. Beales IL. Efficacy of Helicobacter pylori eradication therapies: a single centre observational study. BMC Gastroenterol 2001;1:7.

6. Beales ILP. Excellent results can be achieved without sensitivity testing. BMJ 2002;325.

7. Kim JJ, Kim YH, Rhee PL, et al. Antibiotic heteroresistance of paired-strains of helicobacter pylori isolated from the antrum and corpus of the same patient and PCRbased RAPID fingerprinting analysis. Korean Helicobacter Upper Gastr Res 2002;2:25-9.

8. Torracchio S, Cellini L, Di Campli E, et al. Role of antimicrobial susceptibility testing on efficacy of triple therapy in Helicobacter pylori eradication. Aliment Pharm Ther 2002;14:1639-43.

9. Lamouliatte H, Mégraud F, Delchier JC, et al. Second-line treatment for failure to eradicate Helicobacter pylori: a randomized trial comparing four treatment strategies. Aliment Pharmacol Ther 2003;18: 791-7. 
10. Molnar B, Szoke D, Ruzsovics A, Tulassay Z. Significantly elevated Helicobacter pylori density and different genotype distribution in erosions as compared with normal biopsy specimen detected by quantitative real-time PCR. Eur J Gastroenterol Hepatol 2008;20:305-13.

11. Gomollón F, Sicilia B, Ducóns JA, et al. Third line treatment for Helicobacter pylori: a prospective, culture-guided study in peptic ulcer patients. Aliment Pharmacol Ther 2000;14:1335-8.

12. Gisbert JP, Pajares JM. Helicobacter pylori "Rescue" therapy after failure of two eradication treatments. Helicobacter 2005;10: 363-72.

13. Malfertheiner P, Megraud F, O'Morain C, et al. Current concepts in the management of Helicobacter pylori infection: the Maastricht III Consensus Report. Gut 2007;56: 772-81.

14. Gisbert JP, Gisbert JL, Marcos S, et al. Empirical rescue therapy after Helicobacter pylori treatment failure: a 10 -year single-centre study of 500 patients. Aliment Pharmacol Ther 2008;27:346-54.

15. Lee JM, Breslin NP, Hyde DK, et al. Treatment options for Helicobacter pylori infection when proton pump inhibitorbased triple therapy fails in clinical practice. Aliment Pharmacol Ther 1999;13:48996.

16. Jenks PJ. Causes of eradication of Helicobacter pylori. Antibiotic resistance is the major cause and susceptibility testing may help. BMJ 2002;325:3-4.

17. Parente F, Cucino C, Bianchi Porro G. Treatment options for patients with Helicobacter pylori infection resistant to one or more eradication attempts. Dig Liv Dis 2003;35:523-8.

18. Ferrero M, Ducóns JA, Sicilia B, et al. Factors affecting the variation in antibiotic resistance of Helicobacter pylori over 3year period. Int J Antimicrob Agents 2000; 6:245-8.

19. Miwa H, Nagahara A, Kurosawa A, et al. Is antimicrobial susceptibility testing necessary before second-line treatment for Helicobacter pylori infection? Aliment Pharmacol Ther 2003;17:1545-51.

20. Ikezawa K, Kashimura H, Kojima M, et al. Pretreatment antimicrobial susceptibilities of paired gastric Helicobacter pylori isolates: antrum versus corpus. Helico bacter 2001;4:218-21.

21. Matteo MJ, Granados G, Olmos M, et al. Helicobacter pylori amoxicillin heteroresistance due to point mutations in PBP-1A in isogenic isolates. J Antimicrob Chemother 2008;61:474-7.

22. Andrews J. Disk diffusion method for antimicrobial susceptibility testing. BSAC. V. 2.1.4. Birmingham, UK: British Society for Antimicrobial Chemotherapy 2003.

23. Mishra KK, Srivastava S, Garg A, Ayyagari A. Antibiotic susceptibility of Helicobacter pylori clinical isolates: comparative evaluation of disk-diffusion and E-test methods. Curr Micriobiol 2006;53:329-43.

24. Tankovic J, Lascols C, Sculo Q, et al. Single and double mutations in gyrA but not in gyrB are associated with low- and highlevel fluoroquinolone resistance in Helicobacter pylori. Antimicrob Agents Chemother 2003;47:3942-4.

25. Yahav J, Samra Z, Niv Y, et al. Susceptibil ity-guided vs. empiric retreatment of Helicobacter pylori infection after treatment failure. Dig Dis Sci 2006;51:2316-21.

26. Elviss NC, Owen RJ, Breathnach A, et al. Helicobacter pylori antibiotic-resistance patterns and risk factors in adult dyspeptic patients from ethnically diverse populations in central and south London during 2000. J Med Microbiol 2005;54:567-74.

27. Owen RJ, Xerry J, Peters TM, eare EL. Surveillance and clinical relevance of vacA genotypes of Helicobacter pylori infecting dyspeptic patients in mid-Essex. Commun Dis Public Health 2002;5:106-11.

28. Tiwari SK, Khan AA, Manoj G, et al. A simple multiplex PCR assay for diagnosing virulent Helicobacter pylori infection in human gastric biopsy specimens from subjects with gastric carcinoma and other gastro-duodenal diseases. J Appl Microbiol 2007;103:2353-60.

29. Franceschi F, Severi C, Zullo A, Documento di indirizzo SIGE-AIG0_SIED Sezione Lazio, 2009.

30. Neri M, Milano A, Laterza F, et al. Role of antibiotic sensitivity testing before firstline Helicobacter pylori eradication treatments. Aliment Pharmacol Ther 2003;18: 821-7.

31. Romano M, Marmo R, Cuomo A, et al. Pretreatment antimicrobial susceptibility testing is cost-saving in the eradication of Helicobacter pylori. Clin Gastroenterol Hepatol 2003;1:273-8.

32. Romano M, Iovene MR, Russo MI, et al. Failure of first-line eradication treatment significantly increases prevalence of antimicrobial-resistant Helicobacter pylori clinical isolates. J Clin Pathol 2008;61: 1112-5.

33. Yilmaz 0, Demiray E, Tümer S, et al. Detection of Helicobacter pylori and determination of Clarithromycin susceptibility using formalin-fixed, paraffin embedded gastric biopsy specimens by fluorescence in situ hybridization. Helicobacter 2007; 12:136-41.
34 Mégraud F, Lehours P. Relevance of $\mathrm{H}$. pylori resistance to antibiotics. Clin Microbiol 2007;20:280-322.

35. Blaser MJ, Atherton JC. Helicobacter pylori persistence: biology and disease. J Clin Invest 2004;113:321-33.

36. Alikhani MY, Sadeghifard SN, Farajnia S, et al. Evaluation of selective and non selective media for isolation of Helicobacter pylori from gastric biopsy specimens. Pak J Biol Sci 2007;10:4156-9.

37. Al-Sulami A, Al-Kiat HS, Bakker LK, Hunoon H. Primary isolation and detection of Helicobacter pylori from dyspeptic patients: a simple, rapid method. East Mediterr Health J 2008;14:268-76.

38. Gisbert JP, Bermejo F, Castro-Fernández $\mathrm{M}$, et al. Second line rescue therapy with levofloxacin after $\mathrm{H}$. pylori treatment failure: a Spanish Multicenter Study of 300 patients. Am J Gastroenterol 2008; 103:716.

39. Gisbert JP, Pajares JM. Helicobacter pylori "rescue" regimen when proton pump inhibitor-based triple therapies fail. Aliment Pharmacol Ther 2002;16:1047-57.

40. Peitz U, Sulliga M, Wolle K, et al. High rate of post-therapeutic resistance after failure of macrolide-nitromidazole triple to cure Helicobacter pylori: impact of two secondline therapies in a randomised study. Aliment Pharmacol Therapeut 2002;16: 313-24.

41. Dore MP, Leandro G, Realdi GL, et al. Effect of pretreatment antibiotic resistance to metronidazole and clarithromycin on outcome of Helicobacter pylori therapy. Dig Dis Sci 2000;45:68-76.

42. Glupczynski Y, Mégraud F, Lopez-Brea M, Andersen LP. European multicentre survey of in vitro antimicrobial resistance in Helicobacter pylori. Eur J Clin Microbiol Infect Dis 2001;20:820-3.

43. Kist M, Glocker E. ResiNet-a nationwide German sentinel study for surveillance and analysis of antimicrobial resistance in Helicobacter pylori. Eurosurveillance 2004; 9:44-5.

44. Amitrano M, Spezzaferro M, Sacco F, et al. H. pylori isolates from proximal and distal stomach of patients with $\mathrm{H}$. pylori infection exhibit resistance and sensitivity to the same antibiotic. Proceedings of the 14th National Congress of Digestive Diseases, Rimini, Italy, 2008.

45. Matteo MJ, Granados G, Pérez CV, et al. Helicobacter pylori cag pathogenicity island genotype diversity, within the gastric niche of a single host. J Med Microbiol 2007;56:664-9. 